\title{
Real-time Protein Aggregation Monitoring with a Bloch Surface Wave- based Approach
}

\author{
Sara Santi ${ }^{*}, \mathrm{~b}$, Elsie Barakat ${ }^{\mathrm{a}}$, Emiliano Descrovi ${ }^{\mathrm{c}}$, Reinhard Neier ${ }^{\mathrm{b}}$, Hans Peter Herzig ${ }^{\mathrm{a}}$ \\ ${ }^{a}$ École Polytechnique Fédérale de Lausanne (EPFL), Optics \& Photonics Technology Laboratory \\ (OPT), Rue de la Maladiere 71b, 2002 Neuchâtel, Switzerland; ${ }^{b}$ Université de Neuchâtel, Dept. of

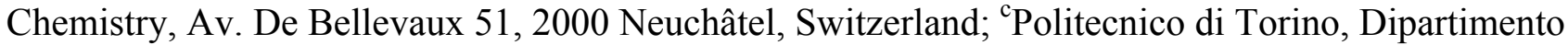 \\ di Scienza Applicata e Tecnologia, C.so Duca degli Abruzzi 24, 10129 Torino, Italy
}

\begin{abstract}
The misfolding and aggregation of amyloid proteins has been associated with incurable diseases such as Alzheimer's or Parkinson's disease. In the specific case of Alzheimer's disease, recent studies have shown that cell toxicity is caused by soluble oligomeric forms of aggregates appearing in the early stages of aggregation, rather than by insoluble fibrils. Research on new strategies of diagnosis is imperative to detect the disease prior to the onset of clinical symptoms. Here, we propose the use of an optical method for protein aggregation dynamic studies using a Bloch surface wave based approach. A one dimension photonic crystal made of a periodic stack of silicon oxide and silicon nitride layers is used to excite a Bloch surface wave, which is sensitive to variation of the refractive index of an aqueous solution. The aim is to detect the early dynamic events of protein aggregation and fibrillogenesis of the amyloid-beta peptide $A \beta 42$, which plays a central role in the onset of the Alzheimer's disease. The detection principle relies on the refractive index changes caused by the depletion of the A 342 monomer concentration during oligomerization and fibrillization. We demonstrate the efficacy of the Bloch surface wave approach by monitoring in real-time the first crucial steps of $A \beta 42$ oligomerization.
\end{abstract}

Keywords: aggregation, Alzheimer's disease, amyloid beta-peptides, biosensing, photonic crystals

\section{INTRODUCTION}

Predicting the three-dimensional configuration of a protein, the so-called protein-folding problem, is one of the most challenging topics of structural biology especially since an increasing number of degenerative pathologies result from an incorrect protein folding (or misfolding). Very often, the protein misfolding is associated with the formation of large insoluble proteic aggregates ${ }^{1-3}$. Disorders such as Alzheimer and Parkinson diseases, Huntington's chorea, CreutzfeldtJacob disease, cystic fibrosis, Gaucher's disease, type II diabetes mellitus and the wide group of systemic amyloidosis are associated with aggregation of misfolded polypeptide chains, commonly referred to as amyloid fibrils. Amyloid fibrils, particularly in their prefibrillar forms (oligomeric precursors), can be highly toxic to cells, suggesting their key role in disease pathogenesis. Amyloid fibrils are ordered aggregates of peptide or protein deposits ranging in size from nanometers to microns, with $\beta$-sheet secondary structure ${ }^{4}$. Not only the structure, but also the process of fibril formation is crucial to correlate the presence of amyloid aggregates to the onset of the degenerative disease ${ }^{5}$.

In the specific case of the Alzheimer's disease (AD), the amyloidogenic peptide is known to be the Amyloid- $\beta$ (A $\beta$ ) peptide, which is naturally formed by the cleavage of a neural transmembrane protein ${ }^{6}$. Its aggregation pathway has been extensively studied ${ }^{7-9}$. It is schematically represented in Fig. 1 and described as follows: the monomeric A $\beta$ peptide, under pathogenic conditions, self-assembles to form the first small soluble aggregate, the oligomers. The oligomeric complex rearrange to the prefibrillar assemblies, which are formed by the so-called $\beta$-sheets structures, typical of amyloid aggregates. Prefibrillar assemblies are hence the building block to the formation of protofilaments, which, as the strings of a rope, are the fibrillar subunit of the mature and insoluble amyloid fibril. A range of experiments, indicates that the fibrils have extensive $\beta$-sheet character, and that these sheets run perpendicular to the fibril axis to generate what is described as a cross- $\beta$ structure ${ }^{10}$. Nevertheless, for many years the only structural information about amyloid fibrils

*sara.santi@epfl.ch; phone: +41 21695 45 46; web: opt.epfl.ch

Biophotonics: Photonic Solutions for Better Health Care IV, edited by Jürgen Popp, Valery V. Tuchin,

Dennis L. Matthews, Francesco S. Pavone, Proc. of SPIE Vol. 9129, 912918 · ( 2014

SPIE $\cdot$ CCC code: $0277-786 X / 14 / \$ 18 \cdot$ doi: $10.1117 / 12.2052842$ 
came from imaging techniques (TEM, AFM) and from X-ray fiber diffraction ${ }^{11}$. Thus far, the real-time monitoring of the $A \beta$ aggregation process is very difficult, if not impossible, with the standard amyloid detection techniques ${ }^{9}$. This problem arises from the fact that those valuable techniques are sensitive to the extent of the mature fibril formation, and mainly silent during the first steps of the oligomerization process. This limitation becomes even more significant if one factors in that oligomers are thought to be the main toxic species, leading to the onset of the AD. In fact, it has been shown ${ }^{12}$ that $\mathrm{A} \beta$ oligomers interact with the membrane of neurons, causing the disruption of ion homeostasis and oxidative stress.

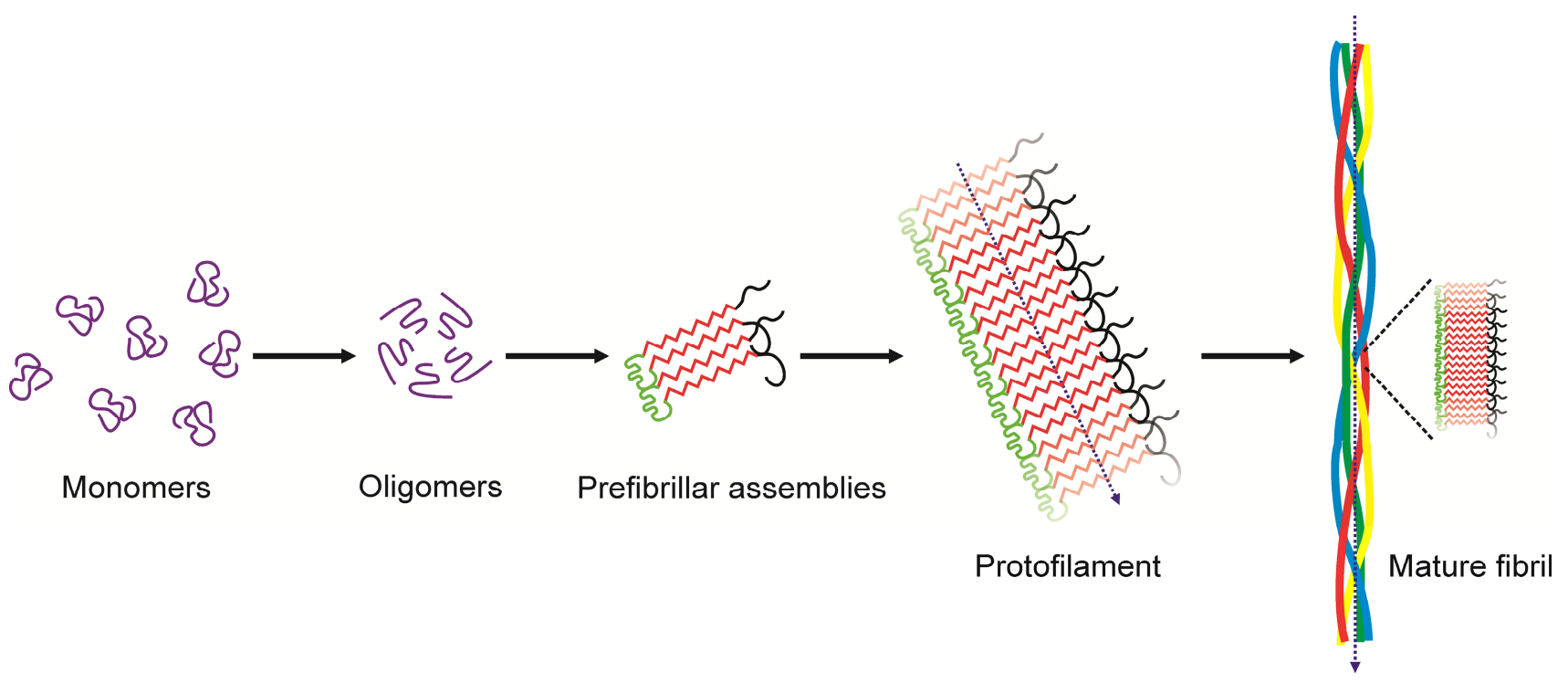

Figure 1. Schematic and simplified amyloid-beta peptide aggregation pathway. At a specific concentration and under pathogenic conditions, monomers can misfold and re-organize to form soluble aggregates commonly known as oligomers. The oligomeric complex rearrange to the prefibrillar assemblies, which are formed by the so-called beta-sheets structures (in red), typical of amyloid aggregates. Two such cross-beta units comprise the protofilament, here represented in a simplified 2D-single layer. The mature fibrils are made by four protofilaments ${ }^{11}$.

In vitro studies on these soluble cytotoxic oligomeric $A \beta$ aggregates should provide a description of the fibril formation mechanism at the very early stages of aggregation ${ }^{13}$. Understanding why and how the peptide may adopt a non-native fold is essential for designing therapeutic approaches ${ }^{14}$. In view of these arguments, we chose to study A $\beta 42$ aggregation. This peptide is the longest of the family of $A \beta$ peptides (42 amino acids) and the most fibrillogenic ${ }^{15}$. We exploit the potential of an innovative sensing approach based on Bloch surface waves (BSWs), to monitor the refractive index changes $(\Delta \mathrm{n})$ of a solution containing an initially monomeric $A \beta 42$ solution. Throughout aggregation, the refractive index properties of the peptidic solution change and it is possible to correlate them to the different steps of $A \beta 42$ fibrillation.

BSW-based sensing has been validated as a sensitive and reliable approach for label-free and real-time biosensing ${ }^{16-18}$. BSWs are Surface Electromagnetic Waves (SEWs) ${ }^{19,20}$. SEWs are non-radiative waves confined at the interface between two media. The optical characteristics of the two media determine the kind of SEW that can be sustained. For example, Surface Plasmon Polaritons (SPP) can be coupled at metal/dielectric interfaces, while Surface Phonon Polaritons can propagate on polar crystals such as $\mathrm{SiC}$ mid-infrared wavelengths. One way to create such a kind of SEW is to use a dielectric multilayer. Since the surface confinement obtained on dielectric multilayers is intimately related to the layers periodicity, this SEW is called Bloch Surface Wave ${ }^{21,22}$. The advantages of BSW, compared to SPP, is the high energy that can be confined at the surface of the multilayer and the larger spectral tunability allowed by the low dispersion of refractive index (n) in the dielectric material (instead of metal for SPP). A very high intensity can be produced at the surface of the multi-layer used for the BSW generation ${ }^{23}$. This property, characteristic of BSW, allows the creation of very sensitive sensors and avoids some of the disadvantages existing with SPR sensors such as the thick sensing region that can decrease the sensitivity. In the case of a BSW-based sensor, the active part is the last layer of the onedimensional photonic crystal which generates the surface wave. In particular, the surface wave can be guided in order to enhance the sensitivity of the sensor ${ }^{24}$. 


\section{EXPERIMENTAL METHOD}

\subsection{Simulations}

The transfer matrix method was used in our simulations. It is based on dividing the grating into $\mathrm{N}$ sections so that each section can be approximately treated as uniform. By applying the appropriate boundary conditions and solving the coupled-mode equations, the transfer matrix gives the relation between the fields at $\mathrm{z}$ and at $\mathrm{z}+\mathrm{L}$ where $\mathrm{L}$ is the total length of the grating ${ }^{22}$.

\subsection{BSW sensing scheme}

In optics, surface waves propagate along the interface between different media. They are solutions of Maxwell's equations. The energy flow is confined on a region that is localized at or near the surface. Bloch surface waves are supported by one dimensional photonic crystals made of alternating layers of transparent materials with different refractive indices (multilayer). They propagate along the interface and the ambient medium leading to a high field enhancement. The amplitude of these waves decay exponentially in the directions both parallel and perpendicular to the boundary with different decay constants. The incident light is coupled under total internal reflection into the surface modes of multilayer only when the propagation constant matching condition is satisfied ${ }^{22}$. The coupling occurs when the projection of the incoming wave vector onto the multilayer interface matches the wave vector of the BSW. In our experiment we work at a fixed wavelength $\lambda(632.8 \mathrm{~nm})$, the coupling is achievable only at a specific angle, which is called the resonance angle. An intensity dip in the reflected beam is produced when BSW are coupled ${ }^{25}$. As the multilayer consists of dielectric materials, the associated losses can be made very low leading to narrower reflectance dip as compared to surface plasmons. Another advantage in using BSWs is the possibility of operating within a broad range of wavelengths, by properly designing a suitable multilayered structure. This tunable localized field confinement is particularly attractive for sensing applications ${ }^{17,26}$.

In the optical setup a He-Ne laser is used to illuminate the photonic crystal oil-contacted to a BK7 glass prism. The incident beam is slightly focused, thus multiple optical rays are incident on the multilayer at different angles. The BSW is excited in a Kretschmann configuration, hence the light is then reflected by the multilayer towards the detector, which is CMOS camera. The evanescent tail of the BSW probes the A $\beta 42$ solution. The coupling is observed as an intensity dip in the reflected angular distribution as imaged onto the detecting camera. The sensing fluidic chamber is positioned vertically. This specific optical arrangement has never been reported before by others. The vertical position allows for accomplishing our specific biosensing goal. During A $\beta 42$ aggregation, the insoluble fibrils precipitate away from the surface sensed by the BSW, thus causing a variation in the refractive index of the solution. Thus, the detectable signal is the angular shift of the reflection minimum (in pixel) as the $\Delta \mathrm{n}$ occurs throughout the aggregation process ${ }^{17}$.

\subsection{Multilayer characterization and BSW response calibration}

The dielectric multilayer was designed to work in water $(\mathrm{n}=1.33)$ and to couple the BSW with a TE-polarized laser working at $\lambda=632.8 \mathrm{~nm}$. The structure is composed of 4 alternating periods constituted by high (H) and low (L) refractive index dielectric materials, according to the scheme: glass-(H-L)x4-H'-water, depicted in Figure 2. Thicknesses and refractive indices of the materials are reported in Table 1. The dielectric layers are deposited on a glass substrate with an Oxford Plasmalab 80+ PECVD machine ${ }^{27}$. 


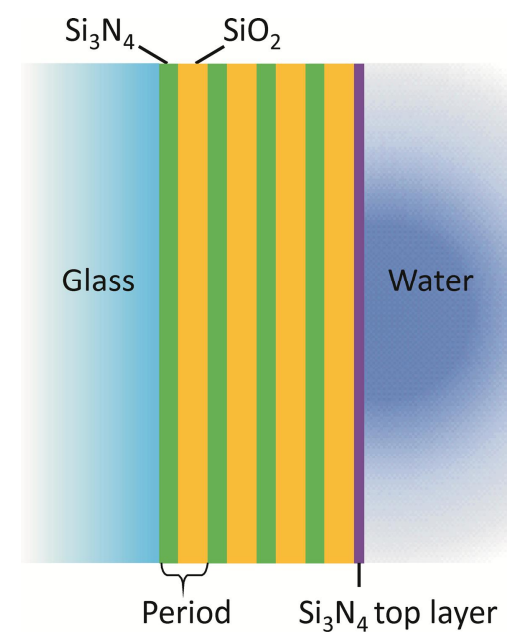

Figure 2. Multilayer design representation, according to the scheme: glass-(H-L)x4-H'-water, where $\mathrm{H}$ is silicon nitride, $\mathrm{Si}_{3} \mathrm{~N}_{4}, \mathrm{~L}$ is silicon oxide, $\mathrm{SiO}_{2}$, and $\mathrm{H}^{\prime}$ is the silicon nitride top layer, which is in contact with the aqueous analyte.

Table 1. Thicknesses and refractive indices of the materials used in the deposition of the one-dimension photonic crystal multilayer.

\begin{tabular}{|c|c|c|}
\hline Material & Layer Thickness & Refractive Index (n) \\
\hline Silicon Nitride (H) & $130 \mathrm{~nm}$ & 1.95 \\
\hline Silicon Oxide (L) & $175 \mathrm{~nm}$ & 1.46 \\
\hline Silicon Nitride (H') & $30 \mathrm{~nm}$ & 1.95 \\
\hline
\end{tabular}

For the calibration of the multilayer sensitivity, two methods were used. In the first, the angular shift of the BSW resonance dip was obtained varying the temperature of d.i. water (resistivity $13 \mathrm{M} \Omega / \mathrm{cm}$ ). Refractive indices of water at different temperature and at $\lambda=632.8 \mathrm{~nm}$ were chosen as reported elsewhere ${ }^{28}$. We decided to work in the linear regime of refractive index variation, which occurs between 40 and $60^{\circ} \mathrm{C}$. In the second method, glucose solutions at different concentration $(\% \mathrm{~m} / \mathrm{v})$ were used. We used the following concentrations: $5 \cdot 10^{-4} \%, 1 \cdot 10^{-3} \%, 5 \cdot 10^{-3} \%, 1 \cdot 10^{-2} \%, 5 \cdot 10^{-2} \%$, $0.1 \%, 0.5 \%, 1.25 \%, 2.5 \%$, and $5 \%$. This method is fully described by Giorgis et $\mathrm{al}^{29}$, where the refractive indices of different glucose solutions are reported. To calculate the sensitivity of the multilayer, an experimentally determined ${ }^{30}$ conversion factor of $0.00107^{\circ} /$ pixels was used.

\subsection{Aß42 sample preparation}

A $\beta 42$ sample was prepared according to a precise protocol described elsewhere ${ }^{31}$. The resulting sample contained a purified monomeric $\mathrm{A} \beta 42$ peptide in a buffered $10 \mathrm{mM}$ Tris $\mathrm{HCl}$ solution at a $\mathrm{pH}$ of 7.5 . The final concentration of the sample was $14 \mu \mathrm{M}$ as determined by UV absorption at $280 \mathrm{~nm}$, using a molar extinction coefficient $\varepsilon$ of $1490 \mathrm{M}^{-1} \mathrm{~cm}^{-1} 32$.

\section{RESULTS AND DISCUSSION}

In order to predict the sensitivity of the BSW supported by the one-dimension photonic crystal described above, we performed the simulation of the BSW response upon a precise refractive index variation of the aqueous medium. Specifically, the BSW intensity dip is resulting from the satisfaction of the coupling conditions, which in our case are $\lambda=$ $632.8 \mathrm{~nm}$, and $\theta=64.075$. When perturbing the medium of a $\Delta \mathrm{n}=1 \cdot 10^{-3}$, the BSW dip is shifted towards a larger $\theta$, equal to 64.125. This is shown in Figure 3. Hence, it is possible to theoretically determine the sensitivity: $\mathrm{S}_{\mathrm{th}}=50^{\circ} / \mathrm{RIU}$. 


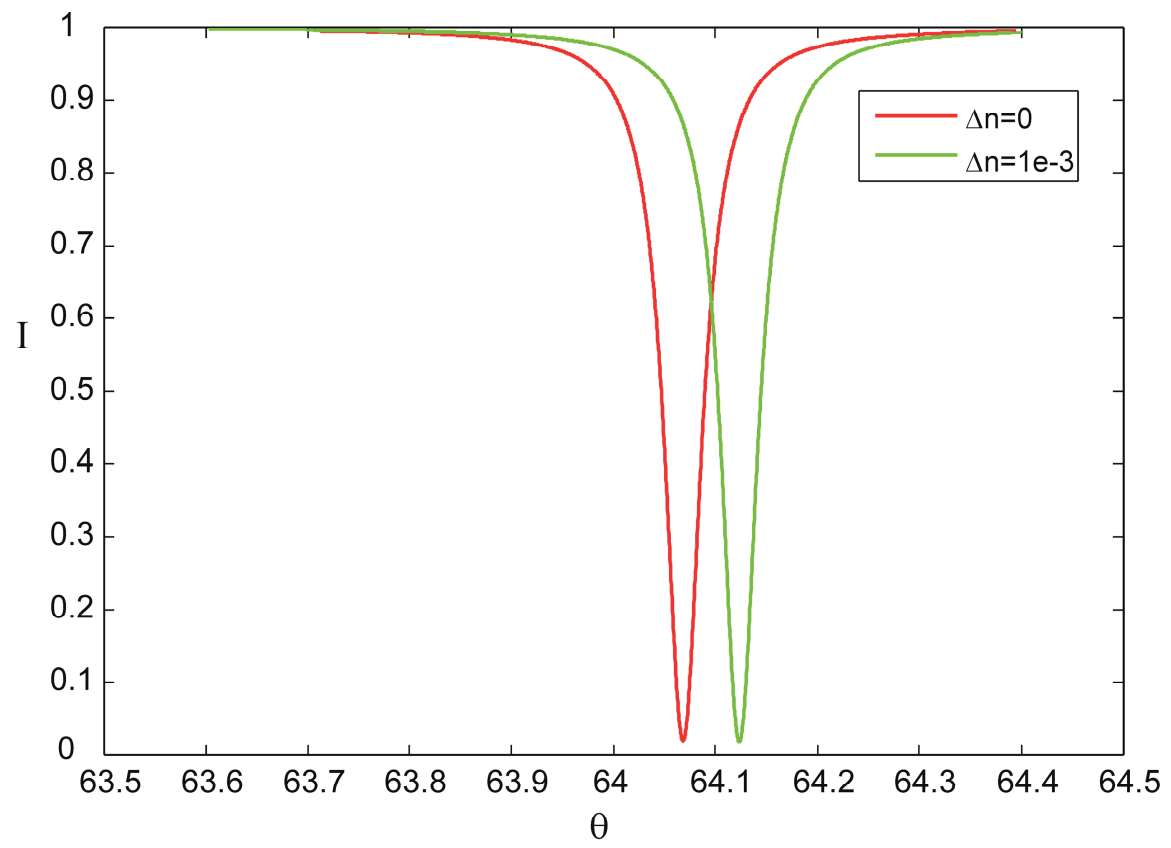

Figure 3. Simulated angular shift of the BSW intensity dip upon a perturbation of the aqueous medium refractive index of $1 \cdot 10^{-3}$.

An experimental calibration of the response of the BSW dip shift was performed according to two different procedures, as described in the "Experimental Methods" section. The first one consisted in varying the temperature of a d.i. water sample, and hence its refractive index, and to follow the shift of the BSW dip position over the pixels of the detector. The result is presented in Fig. 4 and the relationship is linear, as predicted by known formulas ${ }^{29}$. From the slopes of the linear fit, it is possible to estimate a sensitivity of 23174 pixel / RIU. The same calibration experiment was conducted using solutions at different $\%$ in $\mathrm{m} / \mathrm{v}$ of glucose in water. The displacement of the BSW dip over the pixels of the camera was recorded, and the result is presented in Fig.5. As expected, also in this case the relationship between $\Delta \mathrm{n}$ and the pixel shift is linear. The slope of the fit permits the estimation of a sensitivity of 25044 pixel / RIU.

If we consider the experimentally determined conversion factor $0.00107^{\circ} /$ pixel, the sensitivity can be written as: $\mathrm{S}_{\exp }=$ $24.14^{\circ} / \mathrm{RIU}$ and $\mathrm{S}_{\exp }=26.80^{\circ} / \mathrm{RIU}$ for the temperature-based calibration and for the glucose-based calibration, respectively. Those experimental sensitivity values are about the half of the theoretically calculated value, estimated through the simulations presented at the beginning of this section. The main reason for such a difference lie on the fabrication inhomogeneities of the layers in the stack.

The BSW-based approach was then employed to monitor the variation of the refractive index of a solution containing an initially monomeric A $\beta 42$ sample. The sample was obtained as described in the "Experimental Method" section and was then incubated at $37^{\circ} \mathrm{C}$ in the fluidic chamber of the sensor, thus in contact with the multilayer. As explained previously, the sensing chamber is vertically positioned. Hence, during aggregation, the non-soluble mature $\mathrm{A} \beta$ fibrils are precipitating away from the multilayer surface. The BSW then senses a variation of the concentration of the monomeric species present in the solution ${ }^{17}$. The result of this monitoring is presented in Fig.6. It is possible to notice that the refractive index is initially increasing (increase of the pixel shift) and subsequently decreasing (decrease of the pixel shift), with two different descending rates. 


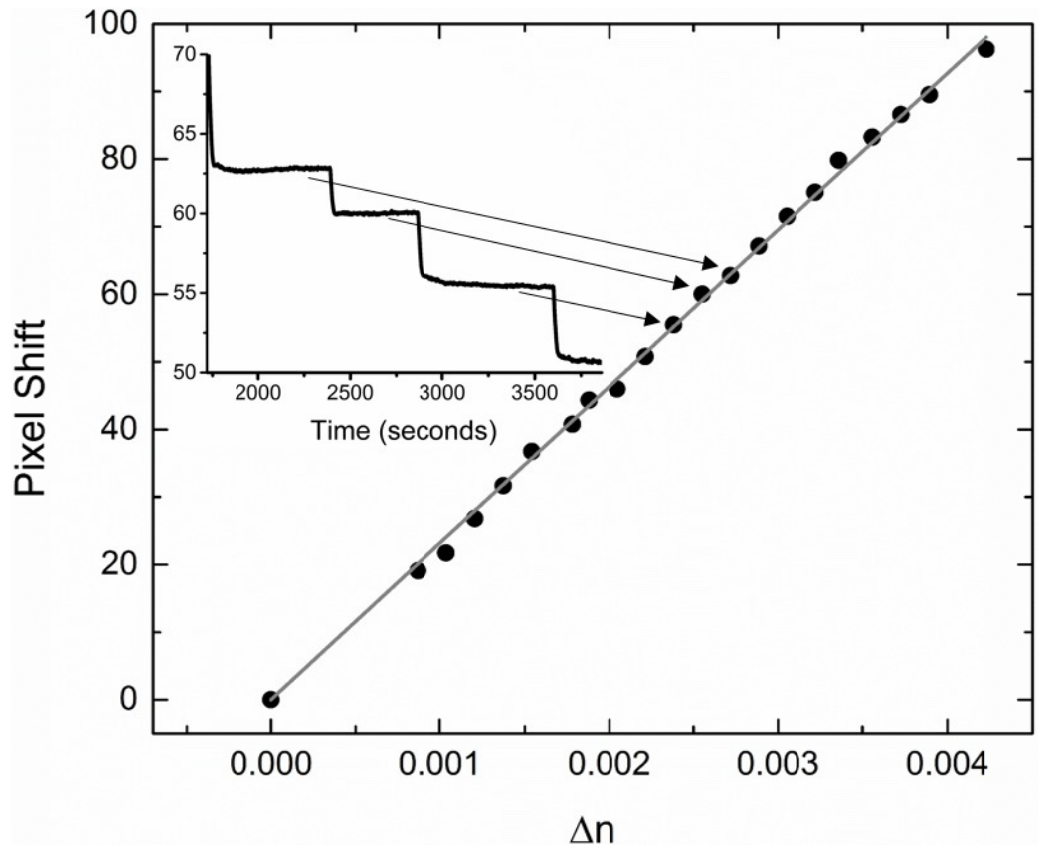

Figure 4. Linear relationship between the module of the refractive index variation $(\Delta \mathrm{n})$ of d.i. water (upon change of temperature) and the shift of the BSW dip position along the pixels of the detector, a CMOS camera. Data were interpolated with a linear fit and the slope of the line represents the sensitivity of the multilayer in pixel / RIU. The temperature of the d.i. water sample was varied from 40 to $60^{\circ} \mathrm{C}$. This temperature-range choice allows for a linear relationship between the variation of the temperature and $\Delta \mathrm{n}$ at $632.8 \mathrm{~nm}$. Inset: sensogram obtained upon the temperature variation of the d.i. water sample, corresponding to the indicated experimental points.

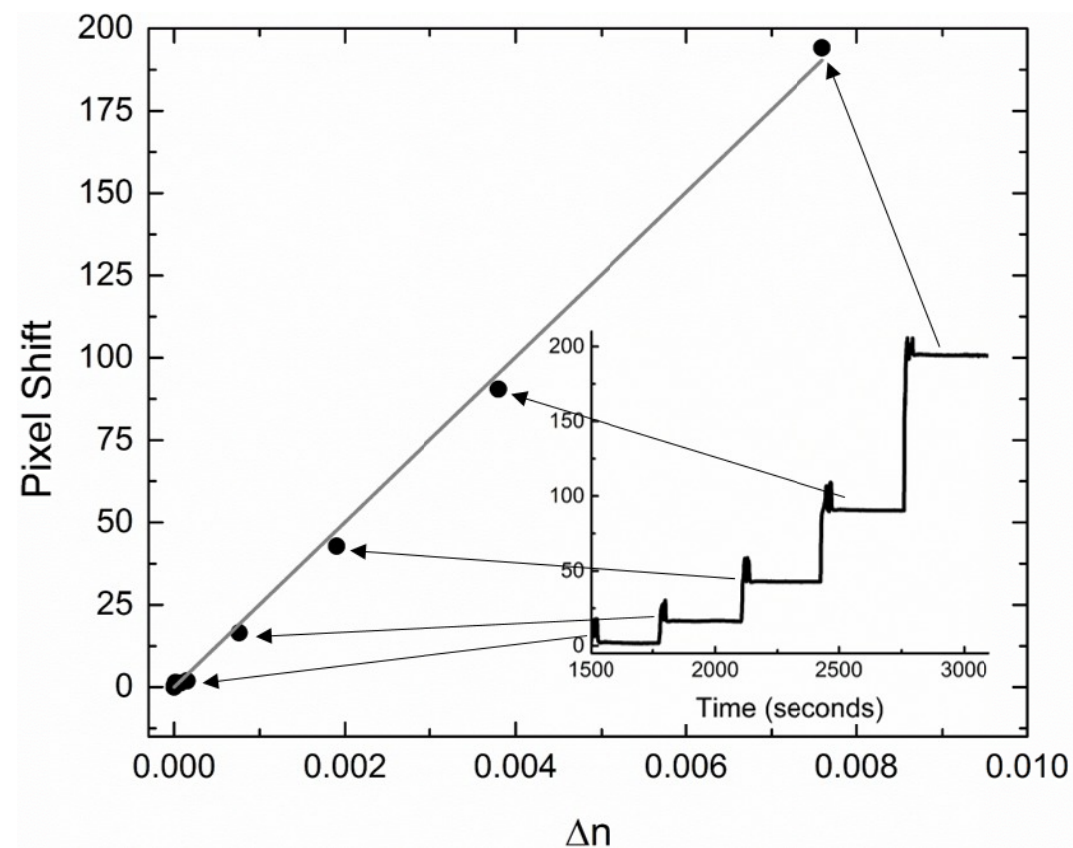

Figure 5. Linear relationship between the variation of the refractive index $(\Delta \mathrm{n})$ of glucose solutions at different concentration $(\% \mathrm{~m} / \mathrm{v})$ in d.i. water and the shift of the BSW dip position along the pixels of the camera. Data were interpolated with a linear fit and the slope of the line represents the sensitivity of the multilayer in pixel / RIU. Inset: sensogram obtained upon the injection of 5 different glucose solutions corresponding to the indicated experimental points. 


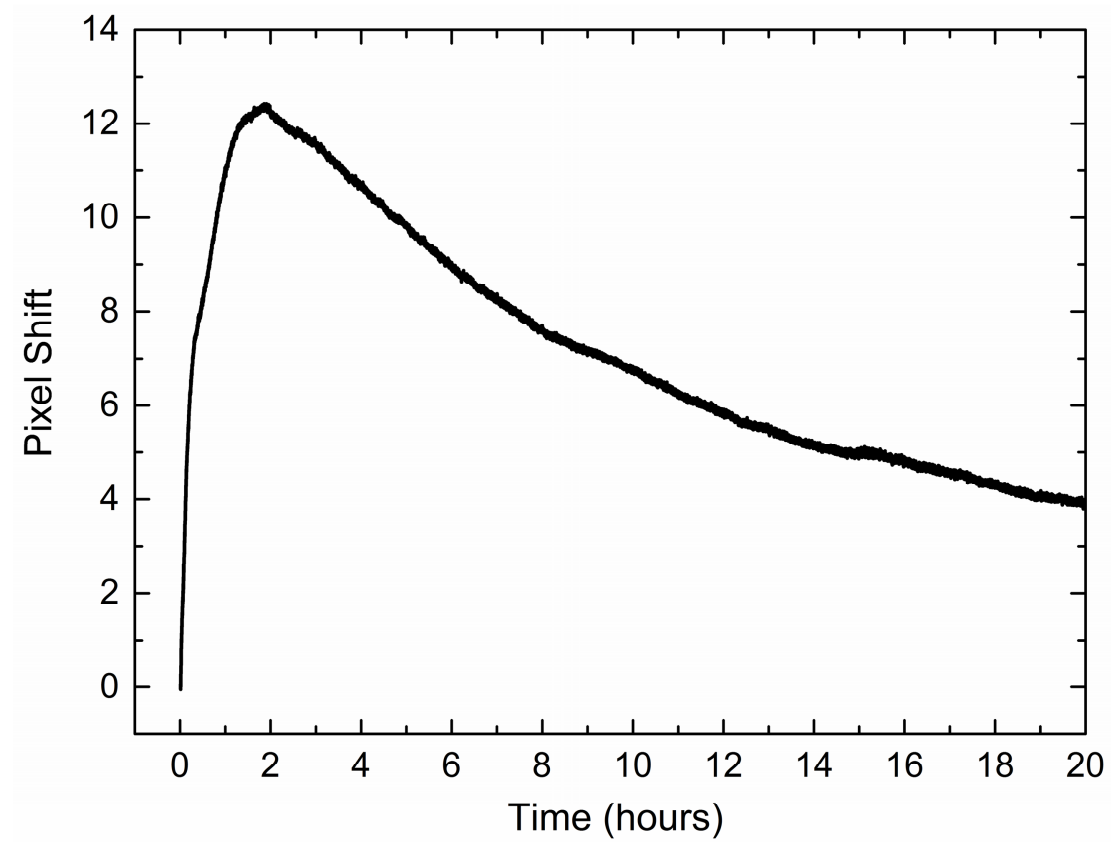

Figure 6. Real-time A $\beta 42$ aggregation monitoring via the BSW-based approach. The refractive index variation $\Delta \mathrm{n}$, in pixel shift, of an initially monomeric A $\beta 42$ peptide solution is displayed against the incubation time. The peptidic sample was incubated at $37^{\circ} \mathrm{C}$, and the initial concentration was $14 \mu \mathrm{M}$ in $10 \mathrm{mM}$ Tris $\mathrm{HCl}, \mathrm{pH}$ 7.5.

When comparing the signal we obtained with results from classical amyloid detection techniques ${ }^{9,31,33}$, one can notice that the increase of the refractive index signal occurs in the same time frame associated with the formation of the first toxic oligomeric species. We identified the increase of the refractive index of the solution with the interaction, or loading, of the $\mathrm{A} \beta$ peptide in an early aggregation state onto the surface of the multilayer. Hence, the refractive index of the solution near the surface of the multilayer, as sensed by the BSW, is higher. In our hypothesis, the descending part of the refractive index variation as a function of time is then correlated to the transformation of the early aggregates into mature and insoluble fibrils, which lose their affinity for the multilayer surface. This explanation is supported by further investigations, as reported elsewhere ${ }^{17}$.

\section{CONCLUSIONS}

This paper reports on a proof of principle of an innovative real-time approach to monitor the aggregation of amyloid peptides. The methodology is based on the use of a one-dimension photonic crystal capable to generate a particular electromagnetic surface wave, called BSW. The results are promising and show the dynamics of the A $\beta 42$ peptide aggregation in a time frame that is silent with other amyloid detection techniques. 


\section{REFERENCES}

[1] Kelly, J. W., "Towards an understanding of amyloidogenesis," Nat Struct Biol, 9(5), 323-5 (2002).

[2] Dobson, C. M., "The structural basis of protein folding and its links with human disease," Philos Trans R Soc Lond B Biol Sci, 356(1406), 133-45 (2001).

[3] Chiti, F. and Dobson, C. M., "Protein misfolding, functional amyloid, and human disease," Annu Rev Biochem, 75, 333-66 (2006).

[4] Glabe, C. G., "Structural classification of toxic amyloid oligomers," J Biol Chem, 283(44), 29639-43 (2008).

[5] Lansbury, P. T. and Lashuel, H. A., "A century-old debate on protein aggregation and neurodegeneration enters the clinic," Nature, 443(7113), 774-9 (2006).

[6] Walsh, D. M., Hartley, D. M., Kusumoto, Y., Fezoui, Y., Condron, M. M., Lomakin, A., Benedek, G. B., Selkoe, D. J. and Teplow, D. B., "Amyloid $\beta$-Protein Fibrillogenesis," Journal of Biological Chemistry, 274(36), 25945-25952 (1999).

[7] Harper, J. D. and Lansbury, P. T., "Models Of Amyloid Seeding In Alzheimer's Disease And Scrapie: Mechanistic Truths and Physiological Consequences of the Time-Dependent Solubility of Amyloid Proteins," Annual Review of Biochemistry, 66(1), 385-407 (1997).

[8] Kelly, J. W., "Mechanisms of amyloidogenesis," Nat Struct Biol, 7(10), 824-6 (2000).

[9] Nilsson, M. R., "Techniques to study amyloid fibril formation in vitro," Methods, 34(1), 151-60 (2004).

[10] Makin, O. S. and Serpell, L. C., "Structures for amyloid fibrils," FEBS J, 272(23), 5950-61 (2005).

[11]Petkova, A. T., Yau, W. M. and Tycko, R., "Experimental constraints on quaternary structure in Alzheimer's beta-amyloid fibrils," Biochemistry, 45(2), 498-512 (2006).

[12] Baglioni, S., Casamenti, F., Bucciantini, M., Luheshi, L. M., Taddei, N., Chiti, F., Dobson, C. M. and Stefani, M., "Prefibrillar amyloid aggregates could be generic toxins in higher organisms," J Neurosci, 26(31), 8160-7 (2006).

[13] Jan, A. and Lashuel, H., [Establishing the Links Between A $\beta$ Aggregation and Cytotoxicity In Vitro Using Biophysical Approaches] Humana Press, 227-243 (2012).

[14] Kroth, H., Ansaloni, A., Varisco, Y., Jan, A., Sreenivasachary, N., Rezaei-Ghaleh, N., Giriens, V., Lohmann, S., López-Deber, M. P., Adolfsson, O., Pihlgren, M., Paganetti, P., Froestl, W., Nagel-Steger, L., Willbold, D., Schrader, T., Zweckstetter, M., Pfeifer, A., Lashuel, H. A. and Muhs, A., "Discovery and Structure Activity Relationship of Small Molecule Inhibitors of Toxic $\beta$-Amyloid-42 Fibril Formation," Journal of Biological Chemistry, 287(41), 34786-34800 (2012).

[15] Ahmed, M., Davis, J., Aucoin, D., Sato, T., Ahuja, S., Aimoto, S., Elliott, J. I., Van Nostrand, W. E. and Smith, S. O., "Structural conversion of neurotoxic amyloid-beta1-42 oligomers to fibrils," Nat Struct Mol Biol, 17(5), 561-567 (2010).

[16] Rivolo, P., Michelotti, F., Frascella, F., Digregorio, G., Mandracci, P., Dominici, L., Giorgis, F. and Descrovi, E., "Real time secondary antibody detection by means of silicon-based multilayers sustaining Bloch surface waves," Sensors and Actuators B: Chemical, 161(1), 1046-1052 (2012).

[17] Santi, S., Musi, V., Descrovi, E., Paeder, V., Di Francesco, J., Hvozdara, L., van der Wal, P., Lashuel, H. A., Pastore, A., Neier, R. and Herzig, H. P., "Real-time Amyloid Aggregation Monitoring with a Photonic Crystalbased Approach," Chemphyschem, 14(15), 3476-3482 (2013).

[18] Descrovi, E., Frascella, F., Ballarini, M., Moi, V., Lamberti, A., Michelotti, F., Giorgis, F. and Pirri, C. F., "Surface label-free sensing by means of a fluorescent multilayered photonic structure," Applied Physics Letters, 101(13), 131105-4 (2012).

[19] Meade, R. D., Brommer, K. D., Rappe, A. M. and Joannopoulos, J. D., "Electromagnetic Bloch waves at the surface of a photonic crystal," Physical Review B, 44(19), 10961-10964 (1991).

[20] Shinn, M. and Robertson, W. M., "Surface plasmon-like sensor based on surface electromagnetic waves in a photonic band-gap material," Sensors and Actuators B: Chemical, 105(2), 360-364 (2005).

[21] Robertson, W. M., "Experimental Measurement of the Effect of Termination on Surface Electromagnetic Waves in One-Dimensional Photonic Bandgap Arrays," J. Lightwave Technol., 17(11), 2013 (1999).

[22] Yeh, P., Yariv, A. and Cho, A. Y., "Optical surface waves in periodic layered media," Applied Physics Letters, 32(2), 104-105 (1978).

[23] Sfez, T., Descrovi, E., Yu, L., Quaglio, M., Dominici, L., Nakagawa, W., Michelotti, F., Giorgis, F. and Herzig, H. P., "Two-dimensional optics on silicon nitride multilayer: Refraction of Bloch surface waves," Applied Physics Letters, 96(15), 151101-3 (2010). 
[24]Descrovi, E., Sfez, T., Dominici, L., Nakagawa, W., Michelotti, F., Giorgis, F. and Herzig, H. P., "Near-field imaging of Bloch surface waves on silicon nitride one-dimensional photonic crystals," Opt Express, 16(8), 5453-64 (2008).

[25]Descrovi, E., Giorgis, F., Dominici, L. and Michelotti, F., "Experimental observation of optical bandgaps for surface electromagnetic waves in a periodically corrugated one-dimensional silicon nitride photonic crystal," Opt Lett, 33(3), 243-5 (2008).

[26] Jamois, C., Li, C., Orobtchouk, R. and Benyattou, T., "Slow Bloch surface wave devices on porous silicon for sensing applications," Photonics and Nanostructures - Fundamentals and Applications, 8(2), $72-77$ (2010).

[27] Paeder, V., Musi, V., Hvozdara, L., Herminjard, S. and Herzig, H. P., "Detection of protein aggregation with a Bloch surface wave based sensor," Sensors and Actuators B: Chemical, 157(1), 260-264 (2011).

[28] Bashkatov, A. N. and Genina, E. A., "Water refractive index in dependence on temperature and wavelength: a simple approximation." Proc. SPIE 5068, 393-395 (2003).

[29] Giorgis, F., Descrovi, E., Summonte, C., Dominici, L. and Michelotti, F., "Experimental determination of the sensitivity of Bloch surface waves based sensors," Opt Express, 18(8), 8087-93 (2010).

[30] Sinibaldi, A., Danz, N., Descrovi, E., Munzert, P., Schulz, U., Sonntag, F., Dominici, L. and Michelotti, F., "Direct comparison of the performance of Bloch surface wave and surface plasmon polariton sensors," Sensors and Actuators B: Chemical, 174(0), 292-298 (2012).

[31]Jan, A., Hartley, D. M. and Lashuel, H. A., "Preparation and characterization of toxic A beta aggregates for structural and functional studies in Alzheimer's disease research," Nature Protocols, 5(6), 1186-1209 (2010).

[32] Gill, S. C. and von Hippel, P. H., "Calculation of protein extinction coefficients from amino acid sequence data," Analytical Biochemistry, 182(2), 319-326 (1989).

[33] Bartolini, M., Naldi, M., Fiori, J., Valle, F., Biscarini, F., Nicolau, D. V. and Andrisano, V., "Kinetic characterization of amyloid-beta 1-42 aggregation with a multimethodological approach," Anal Biochem, 414(2), 215-25 (2011). 